\title{
A QUANTIZATION WATERMARKING TECHNIQUE ROBUST TO LINEAR AND NON-LINEAR VALUMETRIC DISTORTIONS USING A FRACTAL SET OF FLOATING QUANTIZERS
}

\author{
Patrick Bas \\ CNRS, \\ Laboratoire des Images et des Signaux de Grenoble \\ 961 rue de la Houille Blanche Domaine universitaire \\ B.P. 4638402 Saint Martin d'Hères cedex FRANCE \\ Laboratory of Computer and Information Science \\ Helsinki University of Technology \\ P.O. Box 5400 FI-02015 HUT FINLAND
}

\begin{abstract}
This paper presents an extension of the classical Quantization Index Modulation (QIM) data-hiding scheme in the context of valumetric distortions. This scheme uses a fractal quantization structure during the detection but also a content dependent quantization grid to achieve both global constant robustness and the ability to recover the watermark after non-linear valumetric distortions. Previous works are first presented. Then the construction of a floating quantizer that addresses the problem of non-linear transformations is introduced. The embedding and detection schemes for digital image watermarking are afterward introduced, the main characteristic of this scheme is the fact that the detection scheme can use a hierarchical set of quantizers to deal with non-linear valumetric transforms while preserving an average constant quantization step. Finally the performance of this scheme and the comparison with other robust quantization schemes considering valumetric transforms and noise addition are presented.
\end{abstract}

\section{Introduction}

Quantization watermarking techniques ${ }^{1}$, first introduced by Chen and Worwell [1] are widely used in watermarking applications because they provide both robustness to the AWGN channel and high capacity capabilities while preserving the fidelity of the host document. Basically, the Quantization Index Modulation

\footnotetext{
${ }^{1}$ The work described in this paper has been supported (in part) by the European Commission through the IST Programme under Contract IST-2002-507932 ECRYPT and the National French project Fabriano.
} 
(QIM) scheme uses one different quantizer for each code word that is transmitted and the set of quantizer is span on the range of possible values that can be taken by each sample. For example, using a two stages codeword (e.g. a bit of information) requires two disjoints quantizers. The embedding rule of a bit $b$ in a coefficient $C$ is the following:

$$
\begin{aligned}
& \text { If } b[k]=1: C_{w}=2 \Delta \mathcal{E}\left(\frac{C+\Delta / 2}{2 \Delta}\right)-\Delta / 2 \\
& \text { If } b[k]=0: C_{w}=2 \Delta \mathcal{E}\left(\frac{C-\Delta / 2}{2 \Delta}\right)+\Delta / 2
\end{aligned}
$$

Where $\Delta$ represents the distance between one quantized value and the next one, and $\mathcal{E}(x)$ is the integer part of $x$. For security issues, it is also possible to add a key-dependant random signal on the future quantized sample before performing the quantization step and to remove the random signal (call the dither signal) on the quantized value. Chen et. al have first introduced such a method called dither modulation (DM) and have combined it with a distortion compensation module.

However the usage of quantization techniques such as QIM or DM are not straightforward in real application scenarios such as video watermarking where valumetric distortions are often present during the broadcasting process.

\subsection{QIM/DM and valumetric distortions}

A valumetric distortions can be defined by any function $f(p)$ that modifies the value of the original pixel $p$. In practical cases, $f()$ can be either linear (e.g. $f(p)=\alpha(p))$ and represents a gain, or non-linear to represents more widely used distortions. For example, the gamma correction function $\Gamma_{\gamma}(p)$, which has to be especially considered in video watermarking applications when the watermarked signal has to suffer DA/AD conversions, is given by:

$$
\Gamma_{\gamma}(p)=p_{M A X}\left(\frac{p}{p_{M A X}}\right)^{\gamma}
$$

where $p_{M A X}$ is the maximum value of a pixel. A valumetric transform will consequently alter the quantized value in such a way that, if $p_{w}-f\left(p_{w}\right)>\Delta / 2$, the decoding step will probably lead to an error. Such an effect is depicted on Fig. 1 which outlines the displacement of the different quantized values after linear and non-linear valumetric transforms.

\subsection{Previous works}

The goal of this subsection is to present a brief overview of the other works that address the problem of QIM/DM detection in presence of valumetric distortions.

Histogram analysis: Eggers et. al have presented a scheme to address the case of an affine valumetric distortion $f(p)=\alpha p+\beta$ [2]. This method is based 


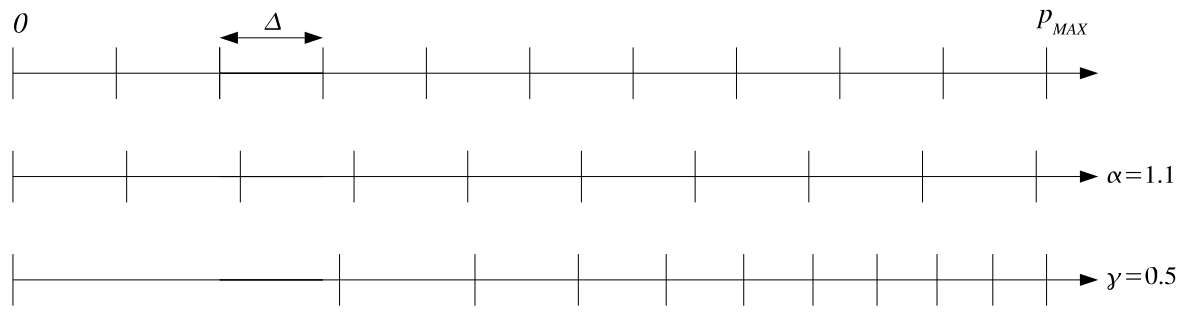

Fig. 1. Effects of valumetric transforms on quantized values: the initial quantization grid (first axis) cannot be used to decode the watermark neither after a linear transform (second axis) nor after a non linear transform such as the Gamma transform (third axis). In many cases, the decoding will lead to erroneous results.

on the analysis of a two-dimentional probability density function (pdf) that depends on the received sample $r$ and the range of the possible deviations for one secret key $k$. The resulting pdf presents periodical components for each deviation and the period is proportional to $\alpha \Delta$. Consequently the authors propose to apply a DFT and a phase shift function for each deviation of the two-dimensional PDF before summing the spectra of the pdfs on the $k$ axis. The maximum of the overall spectrum reveals then the term $\alpha$ and the term $\beta$ is derived from the phase at the location of the maxima. In [3] the authors have also addressed the problem of the non-linear gamma transform by trying to invert the gamma transform before the estimation of $\alpha$ and $\beta$ but this method need a full search of the $\gamma$ parameter and consequently increase the complexity of the algorithm.

ML estimator: Lagendijk et. al have proposed a similar approach than the previous one that also uses the Fourier transform of the histogram to estimate $\alpha$ [4]. They also have presented in [4] and [5] an estimation of parameter $\alpha$ that is based on the Maximum Likelihood (M.L.) estimator. The pdf of the watermarked image after scaling and noise addition is first expressed as $f\left(p, \alpha, \sigma_{n}^{2}\right)$ where $\sigma_{n}^{2}$ denotes the noise variance. The estimates $\hat{\alpha}$ and $\hat{\sigma}_{n}^{2}$ parameters are calculated performing a search on the Likelihood function:

$$
\left(\hat{\alpha}, \hat{\sigma}^{2}\right)=\arg \max _{\alpha, \sigma_{n}^{2}} L\left(\alpha, \sigma_{n}^{2}\right)=\arg \max _{\alpha, \sigma_{n}^{2}} \log f_{p}\left(p, \alpha, \sigma_{n}^{2}\right)
$$

It is important to notice that the likelihood function is expressed for an host signal and a noise that have both gaussian distributions. The model of the host signal does not represent typical distributions of natural images. This can be seen as a limitation of this approach. Moreover this approach is based on a fixed valumetric distortion model (in this case linear), and the use of ML estimator for nonlinear functions is not straightforward.

Host proportional embedding: Another way to deal with the problem of valumetric transforms is to use a quantification step $\Delta_{p}$ that is constructed in such a way that it is proportional to a valumetric feature of the signal. Pérez- 
Gonzaález et. al.[6] have proposed to use a function $g(\mathbf{p})$ which has the property that $g\left(\alpha \mathbf{p}_{i}\right)=\alpha g\left(\mathbf{p}_{i}\right)$ as a weighting function of the initial quantization step ${ }^{2}$. This function can be for example the $l_{n}$ vector norm given by:

$$
g\left(\mathbf{p}_{i}\right)=\left(\frac{1}{L} \sum_{j=i-L}^{i-1}\left|p_{j}\right|^{n}\right)^{1 / n}
$$

For example if $n=1$ and $p_{j} \geq 0$ for all $j$, the quantization step is proportional to the mean of the vector $\mathbf{p}_{i}$. If $n=2$, it is then proportional to the standard deviation of $\mathbf{p}_{i}$. The authors give the proof that when $L \rightarrow+\infty$, the presented scheme, called Rational Dither Modulation, is equivalent to the classical dither modulation scheme presented by Eggers. Another similar scheme has been also proposed at the same time by Oostveen et. al[7], in this scheme the proportional function is chosen as the averaging ${ }^{3}$ function $(n=1)$.

As we will see in section 3, host proportional embedding is adapted for linear valumetric functions when $L$ is large enough. Nevertheless the performances of proportional embedding quantization schemes are poor for non-linear transforms beacause the function $g(\mathbf{p})$ has not special properties to cope with a nonlinearity). Additionally for affine transforms, such schemes cannot handle the addition of an offset value. Another important drawback of proportional embedding schemes is the fact that the quantization step $\Delta$ is not constant. If $L \ll+\infty$ and $n=2$ for example, the quantization step is less important in homogeneous areas than in textured areas. This last point can be a serious handicap considering addition of noise because homogeneous areas will be more sensitive to provide detection errors than textured areas.

\section{A floating quantization scheme with a fractal structure}

As we have mentioned previously, the presented schemes have several drawbacks: histogram analysis enables to identify a linear distortion but its extension to non linear distortions is not straightforward, $M L$ estimator depends both on a host model and a distortion model, host proportional embedding is adapted for linear distortion but not to affine or non linear distortions and moreover the quantization step $\Delta_{p}$ is constant only for stationary iid images.

The objectives of the presented scheme are twice, the first is to provide a quantization scheme that is robust both to linear and non-linear distortions and the second is to provide a constant quantization step for each watermarked coefficient, or at least to bound the range of values that can be taken by the quantization step $\Delta$.

In this paper, we propose first to use a floating quantization grid that is based

\footnotetext{
${ }^{2} \mathbf{p}_{i}$ represents a vector composed by $i$ neighbour pixel values that have been centered.

${ }^{3}$ In this work, the authors do not use the absolute value term because they only consider pixel (positive) values otherwise the two schemes can be considered as similar.
} 
on the local features of the host coefficient to provide robustness to both linear and non linear transforms. A nearly constant quantization step is secondly guaranteed using a fractal set of quantizers. These ideas are presented in the following sections.

\subsection{Dealing with non-linear distortions}

A classical solution to cope with a non-linear distortion $f(p)$ is to locally approximate the function $f()$ by a linear function whose the slope is an approximation of the derivate of $f(p)$. To estimate this derivate, we have can use the fact that images are not idd signals but are more often highly correlated. For example adjacent pixels of an image have highly similar values. We choose in this paper the two neighbours of a given pixel because these three values are often highly similar for natural images. The quantization step $\Delta_{f}$ is afterward constructed by taking the minimum $p_{M i n}$ and the maximum pixel $p_{M a x}$ of these three values respectively as the lower and upper bounds of the quantization grid. Because such a grid relies on the host signal and is specific for each pixel, we have named it a floating quantization grid. The embedding of one bit of information is after done quantizing the middle value $p_{M i d}$. Fig.2 depicts the principle of the floating quantizer. For clarity purposes only two quantized values $p_{1}$ and $p_{0}$ are used in this figure. We can notice that if the distorted values $p_{1}^{\prime}$ and $p_{0}^{\prime}$ are reliable approximations of their equivalent linear transformations, the respective approximation errors $\epsilon_{1}$ and $\epsilon_{2}$ are small under the condition that the nonlinearity is not too important.

A floating quantizer has however to deal with several limitations that have to be addressed.

The first one in the fact that the embedding rate is limited to one third of the total number of sample in the host signal. This is due to the fact that three samples are needed to embed one bit.

The second point to adress is the case where $p_{\text {Max }}=p_{\text {Min }}$. It is however easy to solve this issue by increasing $p_{M a x}$ or decreasing $p_{M i n}$ in such a way that $p_{\text {Max }}-p_{\text {Min }}=\Delta_{\text {min }}$.

The last limitation of this proposal is the fact that the resilience against additive noise is not constant because we choose a quantization step $\Delta_{f}$ that is proportional to the range distance $r=p_{M a x}-p_{M i n}$. This fact is by nature contradictory with the essence of a quantization based-scheme which aims to give an equal quantization step for each watermarked coefficient. We have consequently decided to adopt a specific quantization rule $\mathcal{R}$ that is chosen according to the value of the quantization step $r: \Delta_{f}=\mathcal{R}(r)$. This last point is addressed in the next subsection.

\subsection{Using a fractal quantization structure:}

The main idea is to build a set of $N$ hierarchiezed quantizers $Q=\left\{Q_{1}, \ldots, Q_{N}\right\}$ such that it is possible to have a bijection between one quantizer and one specific 


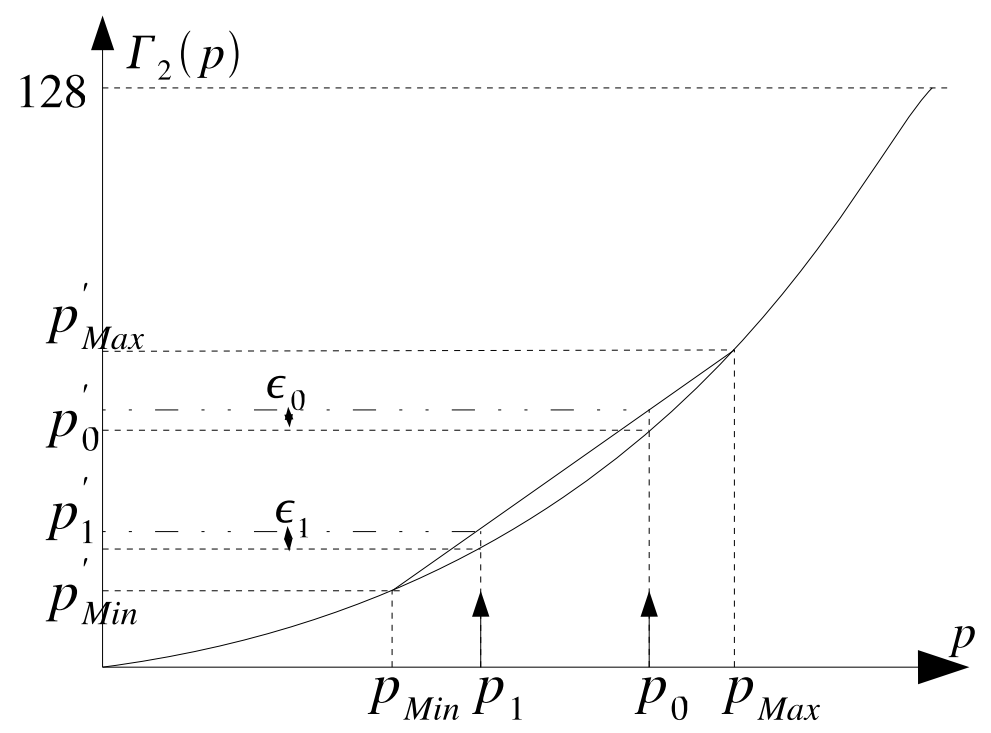

Fig. 2. Effect of a floating quantizer with the function $\Gamma()$ considering $\gamma=2$.

value $r$. In order to have a nearly constant quantization step $\Delta_{f}$ for each quantizer one might fix the minimum and maximum possible values of $\Delta_{f}$ by setting $\alpha_{1} \Delta \leq \Delta_{f} \leq \alpha_{2} \Delta$ where $\alpha_{1} \leq 1, \alpha_{2} \geq 1$ and $\Delta$ is an arbitrary fixed quantization step. To guaranty that there are no overlapping or impossible affectation between on quantizer and its adjacent quantizers these parameters are naturally linked by the relation $\alpha_{2}=2 \alpha_{1}$. The choice of $\alpha_{1}$ depends of different kinds of applications. For example if we want to guarantee a minimum quantization step $\Delta$, this leads to $\alpha_{1}=1$. On the other hand, if we consider an uniform host distribution and want an average quantization step equal to $\Delta$, we then have to choose $\alpha_{1}=0.66$. The value of the floating quantization step $\Delta_{f}$ is given by the relation:

$$
\begin{array}{r}
\Delta_{f}=\frac{r}{2^{N(r)}} \text { if } N(r)>0 \\
\Delta_{f}=\Delta \text { if } N(r)=0
\end{array}
$$

where the power function $N()$ is given using the positive integer part function $\mathcal{E}_{+}():$

$$
N(r)=\mathcal{E}_{+}\left(\log _{2}\left(\frac{r}{\alpha_{1} \Delta}\right)\right)
$$

The calculus of $\Delta_{f}$ naturally leads to the construction of the set of quantizers $Q$ because each different value of $N(r)$ is associated to a number of quantization values. Fig. 3 depicts the set of quantizers which mainly presents a fractal structure: the basic quantization pattern $Q_{1}$ is repeated on $Q_{2}, Q_{3}$ and $Q_{4}$ using a 
contraction factor respectively equal to $1 / 2 ;(1 / 2)^{2}$ and $(1 / 2)^{3}$. Only the quantizer $Q_{0}$ is not a member of the fractal set and this quantizer can be additionally used when initially $p_{M a x}=p_{M i n}$. This fractal structure has the main advantage to avoid to have different quantization cells on the same location on the different axes. Fig. 4 represents the repartition of the set of quantizer $Q$ in function of the range value $r$. We have decided to note each upper border of the quantizer $Q_{i}$ by $q_{i}$. Note also that the quantizer $Q_{0}$ corresponds to the case where $N(r)=0$ and in this case, because the value of $r$ is considered as too small, the quantizer step is forced to be equal to $\Delta$.

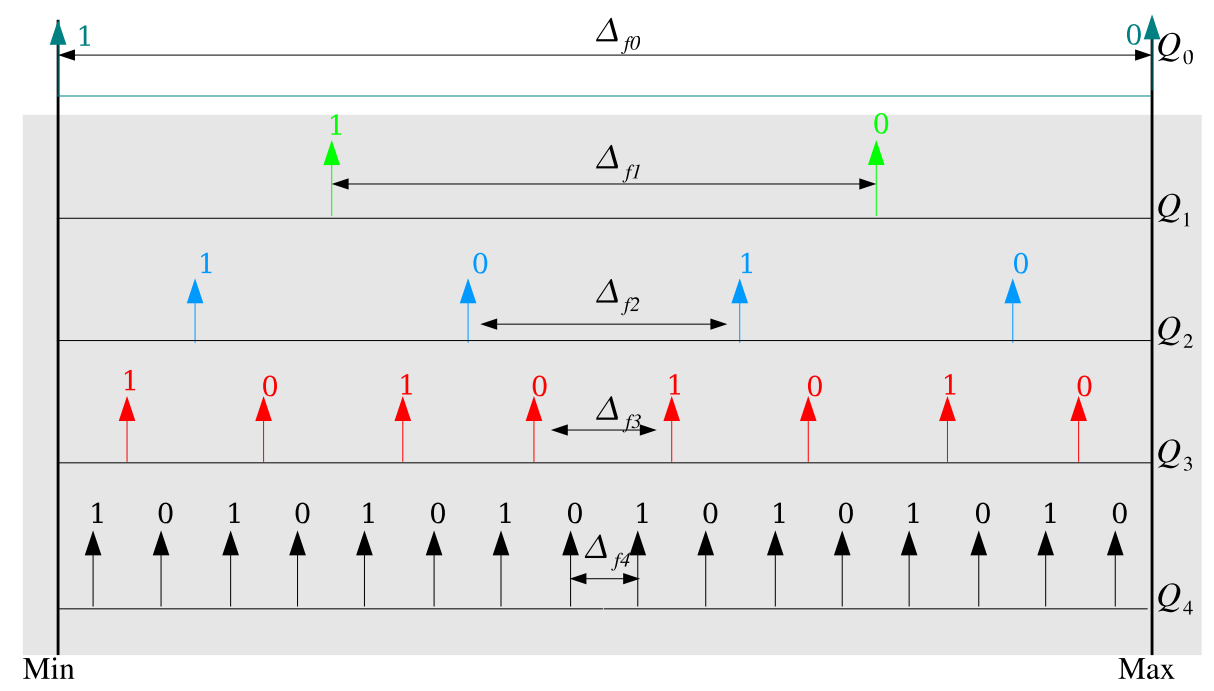

Fig. 3. Building of the set of quantizers. Quantizers that are in the gray area represent a fractal structure. The choice of the quantizer is determined by the value of $r$.

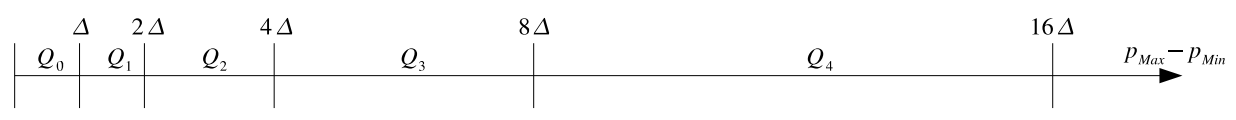

Fig. 4. Repartition of $Q$ in function of $r$ for $\alpha_{1}=1$.

\subsection{Embedding scheme}

Based on the building of the specific floating quantizer, we can afterward apply a classical quantization scheme. The presented scheme is basic but it can be easily improved by using a dither signal or a distortion compensation module 
as proposed by Chen. The embedding of a message $b(k)$ of size $N$ is done by embedding one bit $b$ for each triplet of the original image. The main steps of the embedding procedure are listed below:

- Select a triplet of pixels $\left(p_{i}, p_{j}, p_{k}\right)$. Note that better performance for nonlinear distortion will be obtained if this triplet corresponds to adjacent pixels.

- Order the triplet in $\left(p_{M i n}, p_{M i d}, p_{M a x}\right)$ and compute $r=p_{\text {Max }}-p_{M i n}, \Delta_{f}$ from (2) and (3) that will provide $Q_{i}$.

- If $Q_{i} \neq Q_{0}$ quantize $p_{M i d}$ according to $Q_{i}$ and $b$.

- Else quantize both $p_{M i d}$ and $p_{M i n}$ for $b=0$ or $p_{M i d}$ and $p_{M a x}$ for $b=1$.

- Select another triplet that was not selected before and embed another bit $b$.

\subsection{Detection scheme}

The detection procedure has to consider that the received image has undergone a valumetric transform $f()$. This implies that a given pixel that has been quantized using quantizer $Q_{i}$ may be decoded using another quantizer. To deal with this issue we have first to constrain the possible variations of $f()$ assuming that $\beta_{1} \leq f^{\prime}(p) \leq \beta_{2}$. Assuming that $\left(\beta_{1}, \beta_{2}\right) \in[1 / 2 ; 1] \times[1 ; 2]$, the pixels that are watermarked using a given quantizer $Q_{i}$ may be decoded using $Q_{i}$ and $Q_{i-1}$ or $Q_{i}$ and $Q_{i+1}$. Note that in these particular cases, because we use a set a 2 quantizers, the robustness may be divided by 2 or 4 . Therefore we can also consider another alternate decoding scheme that consists in using only the decoder $Q_{i}$. This is due to the fact that after a valumetric distortion, an initial pixel watermarked using $Q_{i}$ may still be decoded using $Q_{i}$ if the value of $r$ has not changed of quantization cell. The differences between these two decoders will be assessed in the next section. Finally, the different steps of the decoding algorithm are listed below:

- Select $\left(p_{i}, p_{j}, p_{k}\right)$

- Compute $r$ and $\Delta_{f}$.

- Using the fractal detection: if $r>\beta_{1} q_{i-1}$ perform the detection using both $Q_{i}$ and $Q_{i-1}$ and choose the nereast quantization cell considering the two quantizers.

- Using the fractal detection: if $r<\beta_{2} q_{i+1}$ perform the detection using both $Q_{i}$ and $Q_{i+1}$ and choose the nereast quantization cell considering the two quantizers.

- Else or for the classical floating quantizer: perform the detection using $Q_{i}$. 


\section{Results and comparisons}

In this section we outline the capability of the presented scheme to achieve robustness for linear and non-linear distortions and noise addition. We also compare these performances with classical and proportional QIM/DM embedding and detection schemes. In each cases, $1 / 3$ of the pixels are watermarked for both the lena image and baboon image. This is done to achieve the same capacity of embedding. For proportional quantization, we have chosen to use a proportional function that skips the future quantized coefficients to improve the estimation of quantization step. Note that in [6], the authors compute the function $g\left(\mathbf{p}_{i}\right)$ using watermarked samples which is an equivalent way to cope with this problem. We have tested two neighbourhood size: $N=10$ and $N=100$ pixels and we also used $n=2$ which means that the quantization step is proportional to the standard deviation of the selected samples. The floating quantization scheme is tested using both a fractal decoder with $\beta_{1}=0.7$ and $\beta_{2}=1.42$ and a nonfractal decoder.

Fig. 5 and Table. 1 presents the performances of the floating quantization scheme to non-linear distortions (gamma correction and histogram equalization) for different Document to Watermark Ratios.

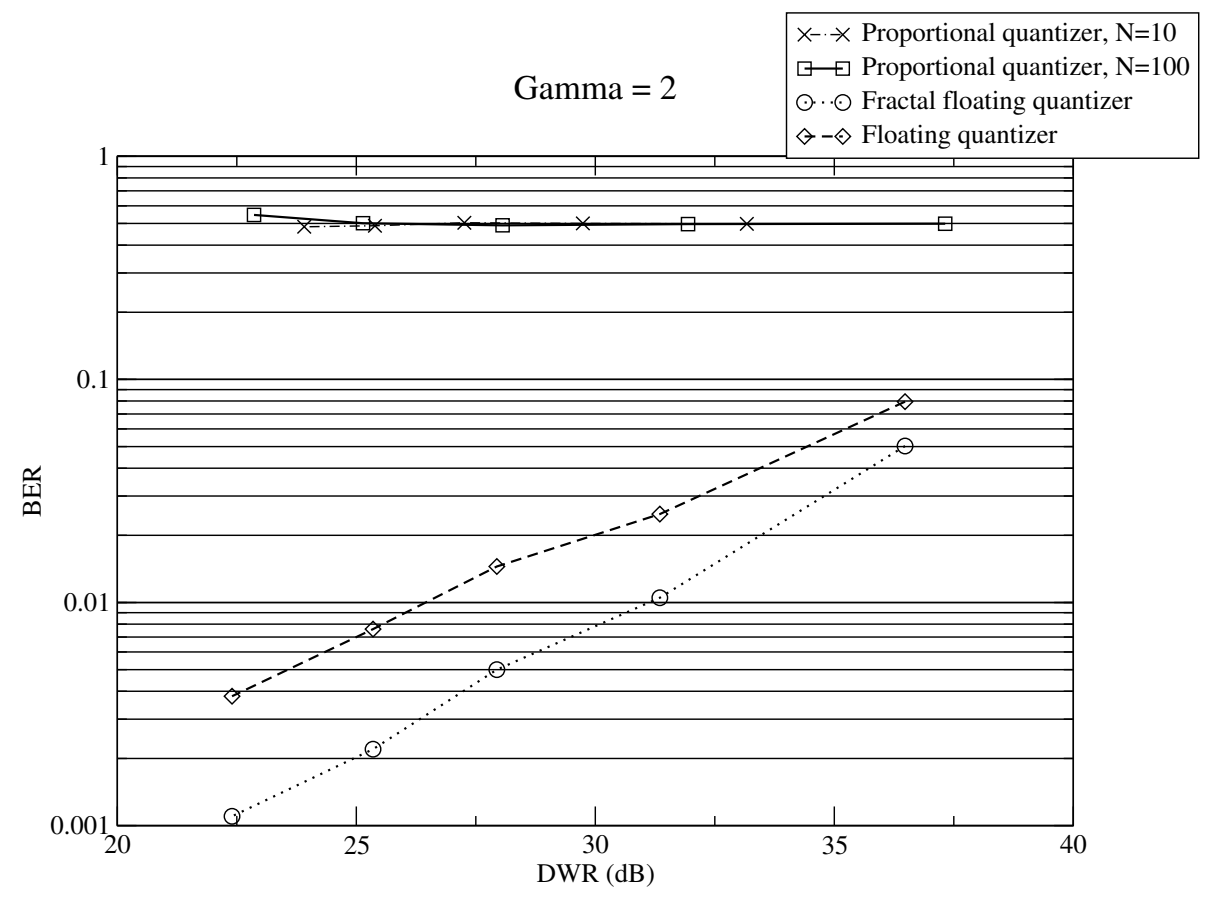

Fig. 5. BER results after gamma correction $\gamma=0.5$ for the lena image. 


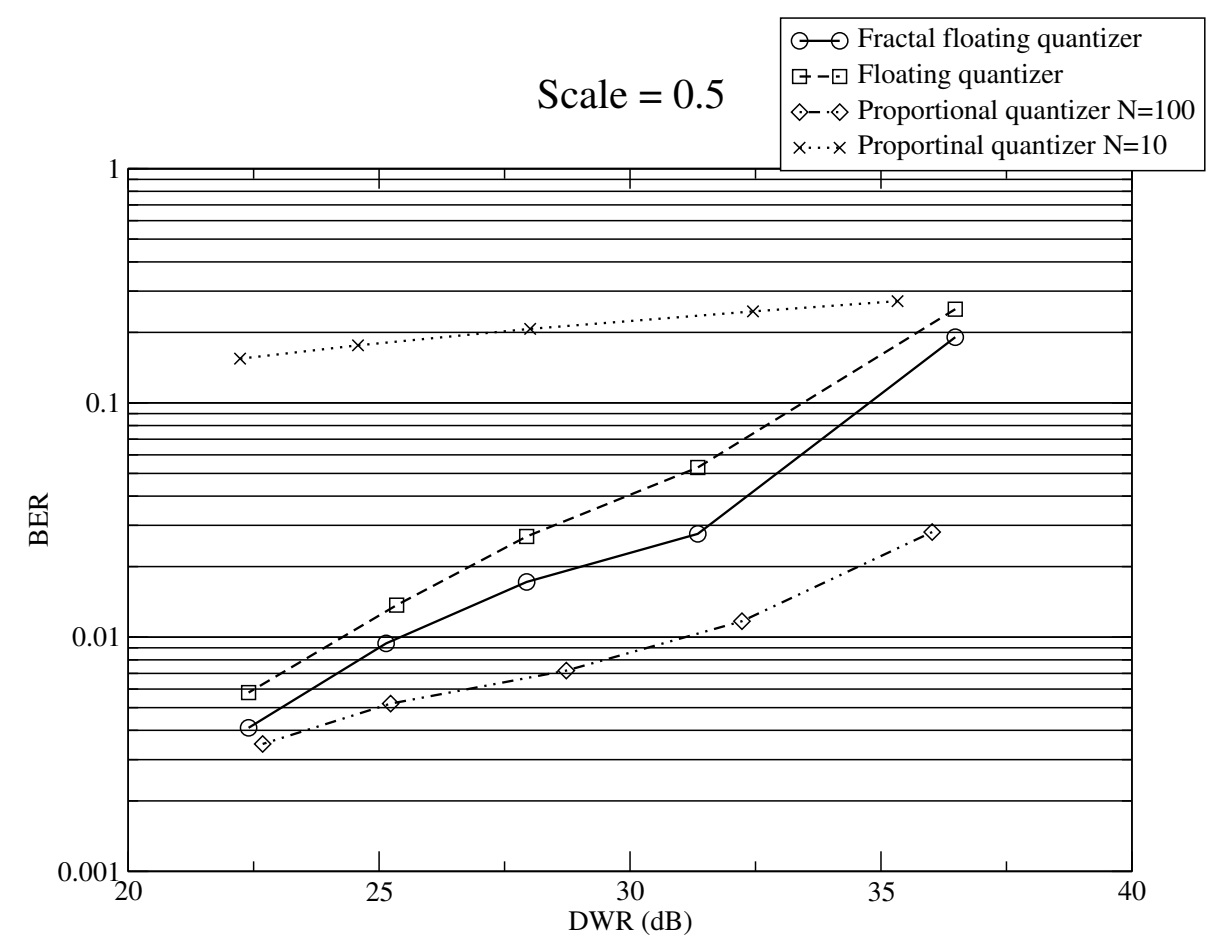

Fig. 6. BER results after linear valumetric transform $\alpha=0.5$ for the lena image.

In Fig. 5, we can notice that the possibility to use multiple quantizers (named in the figure by Fractal quantizer) improve the decoding performances comparing to only one quantizer. Moreover this figure illustrates the fact that the proportional embedding is not adapted to non linear distortions even for little neighbourhood size. In this last case, this is mainly due to the fact that the quantization step of proportional schemes can be too small. For example, if the neighbourhood represents a constant portion of the image, the distance between watermarked samples representing 0 and 1 will be null and will consequenlty lead to decoding errors. The effect of the used valumetric distortion is illustrated in Fig. 8 .

Table 1 presents the robustness of the floating quantization scheme after histogram equalizations that are equivalent to an image dependent nonlinear transform. Fig. 9 represents these functions for the two images and Fig. 8 shows the result on the Lena image. We can notice that the performance of the floating quantization is once again superior to the use of proportional embedding but also depends of the nature of the image. For example, the baboon image, which is more textured than the lena image, offer weaker performance: as mentioned before, this is due to the fact that the linear approximation is not reliable when 


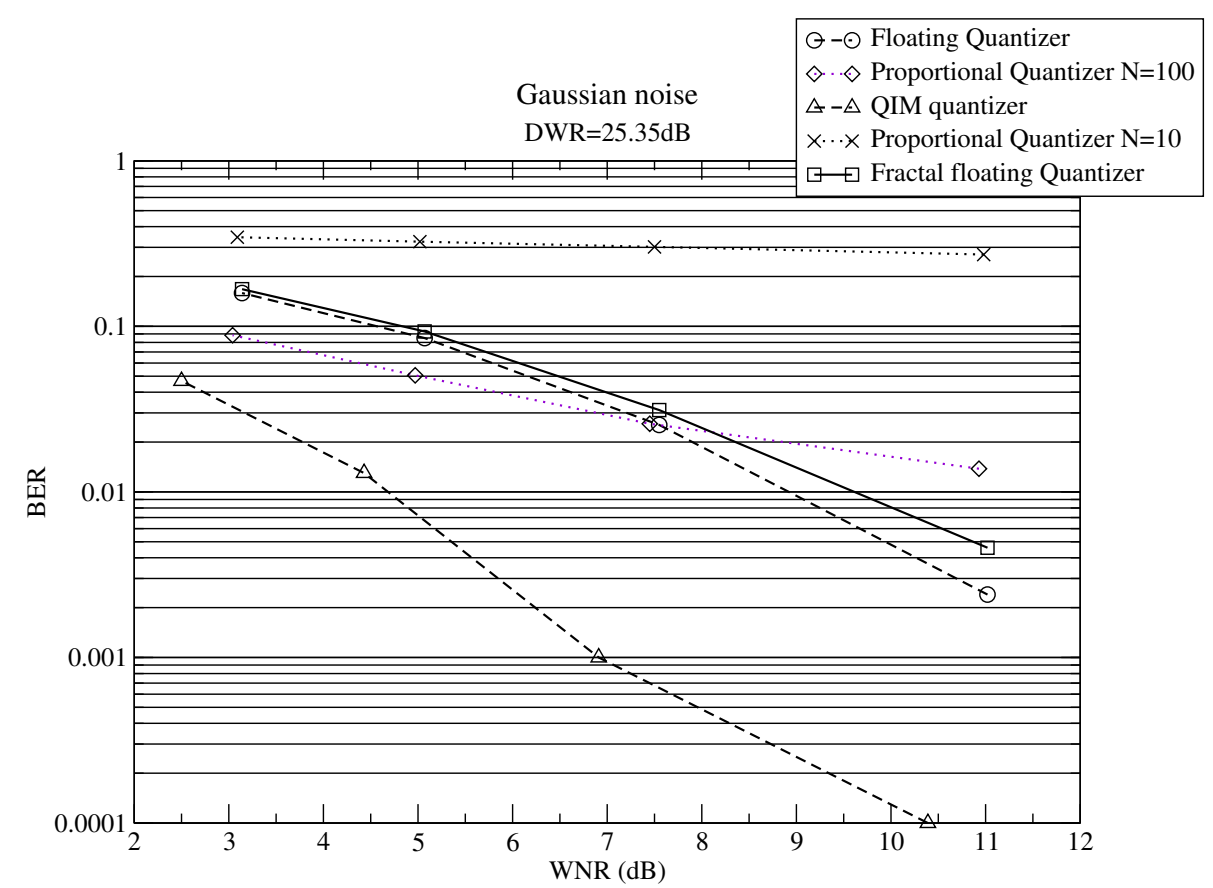

Fig. 7. BER results after gaussian noise addition for the lena image.

$r$ is too important, e.g. when the triplet belong to a textured area.

\begin{tabular}{|r|r|r|r|}
\hline BER (\%) & fractal & non fractal & proportional \\
\hline lena & 3.0 & 3.0 & 36.2 \\
baboon & 12.3 & 15.5 & 58.8 \\
\hline
\end{tabular}

Table 1. BER results after histogram equalization for two different images $(\mathrm{WDR}=27 \mathrm{db}$, neighbourhood size $=200)$.

Fig. 6 presents the performance of the different schemes when the valumetric transform is linear for different $D W R$ s. A scaling factor equal to 0.5 has been applied on the pixels of the watermarked image and no noise is added. Here the proportional quantization scheme can outperform the floating quantization scheme if the size of the neighbourhood is important enough $(N=100)$. Note however that contrary to floating quantization, proportional quantization is robust to an affine transform $f(x)=a x+b$ only when $b=0$.

Fig. 7 compare the robustness after Gaussian noise addition for a $D W R$ equal to $25.35 \mathrm{~dB}$. In this case, we can see that the classical QIM/DM scheme 

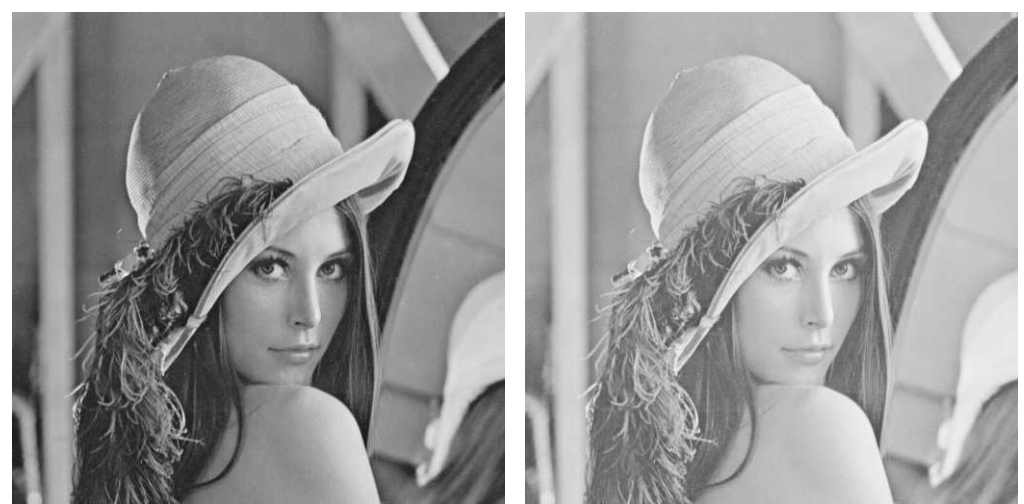

Original lena

lena after Gamma transform $(\gamma=2)$

Fig. 8. Effects of the gamma transform for the presented tests images.

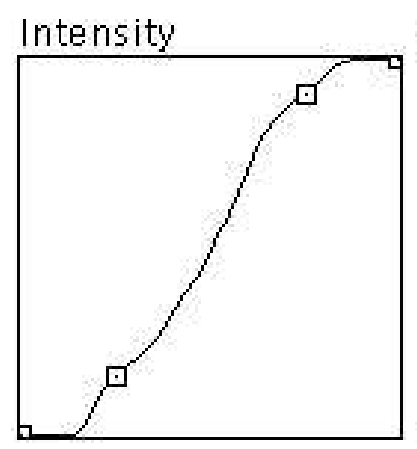

lena
Intensity

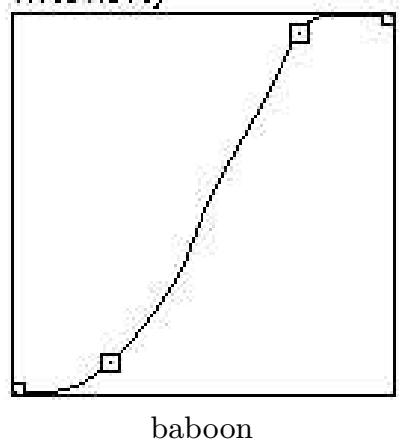

Fig. 9. $f(p)$ for histogram equalization on two images.

outperforms all the other schemes, and that the floating quantization scheme is weaker than the proportional quantization scheme for a large neighbourhood size $(N=100)$. This is due to the fact that $\Delta_{f}$ is proportional to $r$ that is also subject to noise. When the neighbourhood size is not enough important however, the proportional quantizer scheme is less efficient that the floating quantizer because a minimum quantization step is not guaranteed with the first solution.

\section{Concluding remarks and perspectives}

We have presented in this paper a practical implementation of a quantization watermarking scheme that is more robust to non-linear distortions than previous proposed schemes. This performance gain is mainly due to the use of a floating quantizer and the adoption of a fractal set of quantizers.

Future works will address the capacity limitation of this scheme by performing 
an iterative embedding on each sample to achieve a capacity equal to one bit per sample.

\section{References}

1. Chen, B., Wornell, G.W.: Quantization index modulation: A class of provably good methods for digital watermarking and information embedding. IEEE Transactions on Information Theory 47 (2001) 1423-1443

2. Eggers, J.J., Buml, R., Tzschoppe, R., Girod, B.: Scalar costa scheme for information embedding. IEEE Trans. on Signal Processing 51 (2003) 1003-1019

3. Eggers, J.J., Bauml, R., Girod, B.: Estimation of amplitude modifications before scs watermark detection. In: Proceedings of SPIE, Security, Steganography and Watermarking of Multimedia Contents IV, San Jose, USA (2002)

4. Shterev, I., Lagendijk, R., Heusdens, R.: Statistical amplitude scale estimation for quantization-based watermarking. In: Proceedings of SPIE, Security, Steganography and Watermarking of Multimedia Contents VI, San Jose, USA (2004)

5. Lagendijk, R.L., Shterev, I.D.: Estimation of attacker's scale and noise variance for qim-dc watermark embedding. In: Proc. if ICIP, Singapore (2004)

6. Perez-Gonzalez, F., Barni, M., Abrardo, A., Mosquera, C.: Rational dither modulation: a novel data-hiding method robust to value-metric scaling attacks. In: IEEE International Workshop on Multimedia Signal Processing, Siena, Italy (2004)

7. Oostveen, J., Kalker, T., Staring, M.: Adaptive quantization watermarking. In: Proceedings of SPIE, Security, Steganography and Watermarking of Multimedia Contents VI, San Jose, USA (2004) 